\title{
On the Observed Robustness of Disturbance-Observers; A Technical Explanation and Simulation Validation
}

\author{
Michael R. Hannan \\ NASA Marshall Space Flight Center MS: EV41 \\ Huntsville, Alabama
}

Keywords: Disturbance-Observer, DisturbanceAccommodation, Adaptive-Observer,

Disturbance-Inputs, Disturbance-Models

\begin{abstract}
This paper examines the ability of a real-time "disturbance-observer" to adapt-to and closely estimate the time-behavior of a disturbance-input $w(t)$ (and of it's state-vector $z(t)$ ) even when the actual $w(t)$ time-behavior deviates from the observer's "internal-copy" of the nominal/predicted $w(t)$-behavior. By means of technical explanations and confirming simulation studies of numerical examples, the disturbanceobserver's adaptive ability is explained in terms-of the underlying spline-model used to derive the disturbance state-model and the intrinsic dynamic characteristics of a state-observer.
\end{abstract}

\section{Introduction}

The modern-control concept of a "disturbanceobserver" for linear dynamical systems was originally developed in a 1966-68 research-study funded by NASA's Marshall Space-Flight Center through the Huntsville Office of the General Dynamics Corporation [1], [2]. Those earliest results, obtained by variations of the optimal Linear Quadratic Regulator control-design methodology and by a novel alternative, purely (linear) algebraic/ stabilization control-design method $[2 ; \mathrm{pp}$. ], were first published in a series of 1968-1970 journal papers [3]-[5].

The concepts of: (i) a disturbance-"state" $z(t)$, (ii) an associated disturbance state-model $\dot{z}=D z$ [5; p.225] and (iii) a corresponding disturbance-state observer (both full-order and reduced-order) have turned out to comprise a surprisingly-effective tool in the design of "smart", high-performance, modern MIMO control systems [6]. In particular, such control-systems can automatically "accommodate" (i.e., cancel-out, minimize or optimally utilize) the presence of a broad variety of uncertain, persistent, uncontrollable-inputs (disturbance-inputs) $w_{i}(t)$ that would otherwise interfere-with the desired closedloop behavior of a multivariable dynamic system, [7].

In connection with practical applications of disturbance-accommodating control system design many users have observed that a disturbanceobserver is capable of adapting-to, and producing surprisingly-close, real-time estimates $\hat{w}(t)$ of, the actual disturbance time-behavior $w(t) \ldots$. - even when the actual time-behavior of $w(t)$ deviates from the behavior modeled by the "internal-copy" [5; Fig. 2 and text below] of the $w(t)$ state-dynamics embodied in the disturbance-observer design (i.e., in the splinemodel and $D$-matrix).

In this paper a technical explanation, with supporting examples and simulation results, is presented to explain the adaptive/robust disturbanceestimation capability of a disturbance-observer.

\section{Spline-Models and State-Models for Uncertain Disturbance-Inputs}

To minimize the complexity of this presentation, we will restrict attention to the simpler case of a single (and scalar) disturbance-input $w(t)$. However, in principle, all our results apply also to the case of multivariable disturbance-inputs

$$
w(t)=w=\operatorname{col} .\left(w_{1}(t), w_{2}(t), \cdots, w_{p}(t)\right) .
$$

In the mathematical-theory of disturbanceaccommodation, [7], [8], the uncertain time-behavior of a (real-valued, scalar) disturbance-input $w(t)$ is modeled by a spline-function of the form

$$
w(t)=C_{1} f_{1}(t)+C_{2} f_{2}(t)+\cdots+C_{M} f_{M}(t) ; w=\text { scalar, }
$$

where $\left\{C_{i}\right\}_{1}^{M}$ is a set of "arbitrary," real-valued weighting-coefficients that are presumed essentially "constant" but may abruptly change-values in a timesparse, once-in-a-while manner (hereafter referred-to as a "stepwise-constant" manner). In (1) the set $\left\{f_{i}(t)\right\}_{1}^{M}$ is a set of real-valued, well-defined, wellbehaved, (continuously differentiable, etc.) independent, known, "basis-functions" that are userchosen to represent the user's knowledge/ presumptions about the fundamental-modes (building-blocks) of $w(t)$ time-behavior. At each moment " $t$ " the basis-functions are "weighted" and linearly-combined according-to (1) to produce the actual time-behavior (kinematics) of $w(t)$ at that moment.

In practical applications the time-behavior of $w(t)$ is "uncertain" because the values of the "stepwise- 
constant" weighting coefficients $\left\{C_{i}\right\}_{1}^{M}$ in (1) are (typically) unknown, not reliably predictable, and not directly measurable. Moreover, the user's a priori choice of basis-functions $\left\{f_{i}(t)\right\}_{1}^{M}$ might not exactly "match" the actual basis-functions associated with real-life, $w(t)$ time-behavior encountered in a realistic, practical application.

In disturbance-accommodation applications, the practicality and effectiveness of the spline-model (1) is considerably enhanced if the user chooses basis-functions $f_{i}(t)$ in (1) from the class of functions that are of "linear-dynamic" (LD) type, [8; Eqs. 6-12]. That is, each $f_{i}(t)$ obeys a respective well-behaved, well-defined linear, homogenous differential equation (of finite-order). In that case, on those sub-intervals $\Omega$ of time where the $\left\{C_{i}\right\}_{1}^{M}$ all remain constant, the function $w(t)$ defined by (1), with "arbitrary" (constant) weightingcoefficients $\left\{C_{i}\right\}_{1}^{M}$, also satisfies a knowable, linear, homogenous differential equation (of finite-order) of the form

$\frac{d^{\rho \rho} w}{d t^{\rho}}+\alpha_{\rho} \frac{d^{\rho-1} w}{d t^{\rho-1}}+\cdots+\alpha_{2} \frac{d w}{d t}+\alpha_{1} w=0, t \in \Omega$,

where the $\left\{\alpha_{j}\right\}_{1}^{\rho}$ in (2) are known/knowable parameters that depend only on the basis set $\left\{f_{i}(t)\right\}_{1}^{M}$ and, in some cases, may be time-varying parameters, [8]. Consequently, the one, higher-order differential equation disturbance-model (2) can be replaced by an equivalent "disturbance state-model" [6] consisting of a set of " $\rho$ " first-order, ordinary, coupled differential equations of the form

$$
\dot{z}_{i}=g_{i}\left(z_{1}, z_{2}, \cdots z_{\rho}\right), \quad(\cdot) \triangleq ; \quad \rho \geq M,
$$

where the "disturbance state-variables" $z_{i}$ are suitably-defined independent, functions of $w(t)$ and of the first $(\rho-1)$ time-derivatives of $w(t)$. For instance, as one illustration, one can define the $z_{i}$ as the "phasevariables"

$z_{1} \triangleq w, \quad ; \quad z_{2} \triangleq \dot{w} \quad ; \quad z_{3} \triangleq \ddot{w}, \cdots, z_{\rho} \triangleq(\stackrel{(\rho-1)}{w}$,

in which case the disturbance state-model becomes (in light of (2), (4))

$$
\left\{\begin{array}{l}
\dot{z}_{i}=z_{i+1} \quad i=1,2, \cdots,(\rho-1), \\
\dot{z}_{\rho}=-\alpha_{1} z_{1}-\alpha_{2} z_{2}-\cdots-\alpha_{\rho} z_{\rho} .
\end{array}\right.
$$

The results (4), (5) can be more-compactly expressed in the vector-matrix format

$$
\begin{gathered}
w(t)=<h_{o}, z(t)>;<\cdot, \cdot>- \text { denotes inner product } \\
\dot{z}=D_{o} z ; \quad z\left(t_{o}\right)=\text { arbitrary, } t \in \Omega
\end{gathered}
$$

where

$$
\begin{aligned}
& \boldsymbol{h}_{o} \triangleq \operatorname{row}(1,0,0, \cdots, 0) \\
& \boldsymbol{D}_{o} \triangleq\left[\begin{array}{ccccc}
0 & 1 & 0 & \cdots & 0 \\
0 & 0 & 1 & \cdots & 0 \\
\vdots & & & \ddots & \vdots \\
0 & 0 & 0 & \cdots & 1 \\
-\alpha_{1} & -\alpha_{2} & -\alpha_{3} & \cdots & -\alpha_{\rho}
\end{array}\right] .
\end{aligned}
$$

As shown in [6; p.636] the restricted "disturbance state-model" (6) can be easily generalized to correctly represent the time-sparse, unpredictable jumps in the values of the "constants" $C_{i}$ in (1) by adding, to the right side of (6), the $\rho$-vector inputterm $\sigma(t)$ to obtain $\dot{z}=D_{o} z+\sigma(t)$ where

$$
\sigma(t)=\operatorname{col} .\left(\sigma_{1}(t), \sigma_{2}(t) \cdots, \sigma_{\rho}(t)\right)
$$

and where each element $\sigma_{i}(t)$ of $\sigma(t)$ is a time-sparse sequence of uncertain dirac-impulses having unknown intensities and unknown (sparse) arrivaltimes. The effect of the impulse-sequences $\sigma_{i}(t)$ on the solutions $z(t)$ of $(6)$ is to impart uncertain, timesparse jumps in the values of the corresponding $z_{i}(t)$ and thereby "cause/model" similar, corresponding, unknown time-sparse jumps in the values of the $C_{i}$ in (1), [8; p.395].

Since the primary purpose of this paper is to provide a technical explanation for a disturbance observer's apparent, inferred "adaptive-behavior" of the $C_{i}$-values in (1), between successive time-sparse jumps in the $C_{i}$, (i.e. for $t \in \Omega$ ) it suffices to consider the corresponding restricted state-model (6) in the remaining sections of this paper. Moreover, to further simplify things, we will hereafter assume the parameters $\left\{\alpha_{i}\right\}_{1}^{\rho}$ in (2), (6) are all constant (and realvalued).

\section{Real-Time Disturbance-State Observers}

It is recalled that the conversion of the LD splinemodel (1) into a homogenous, linear differential equation model (2) and subsequently into a 
homogenous, linear, "disturbance state-model" (6) is predicated on the tacit assumption that the set of "stepwise-constant" weighting coefficients $\left\{C_{i}\right\}_{1}^{M}$ in (1) remain constant over the time-intervals $t \in \Omega$ being considered. That simplifying assumption allows us to avoid several irrelevant side-issues that would distract from the previously stated primary purpose of this paper.

To develop the results in this paper, we lose no generality (and achieve further reduction of complexity) by employing the simplified, continuoustime, disturbance-state observer introduced-in $[8 ; \mathrm{p}$. $434]$ corresponding to the special (and relatively rare) case where the (scalar) disturbance $w(t)$ is directly, reliably and continuously measurable by some appropriate (noise-free) sensor. For that special case; the "full-order" version of a continuous-time, "disturbance-state observer", as presented in [8] and based on (6), has the simple form [8; Eqs. (52), (53)].

$$
\dot{z}=D_{o} \hat{z}-\boldsymbol{k}_{d o}\left[w(t)-\left\langle\boldsymbol{h}_{o}, \hat{z}(t)\right\rangle\right]
$$

where $w(t)$ in (8) denotes the real-time measurement of the actual disturbance (i.e. the "input" to the stateobserver (8)), and $\boldsymbol{k}_{d o}$ denotes the observer gainvector that must be designed to assure $\hat{z}(t) \rightarrow z(t)$ sufficiently fast for the application requirements. For the latter purpose, the dynamical equation governing the observer-error $e_{z} \triangleq(z-\hat{z})$ is easily computed to be [8; Eq. (53)]

$$
\dot{e}_{z}=\left[D_{o}+k_{d o} h_{o}\right] e_{z},
$$

and thus the gain-vector $\boldsymbol{k}_{d o}$ should be designed to make all solutions $e_{z}(t)$ of (9) approach zero sufficiently fast. Since we are assuming $D_{o}$ is a constant matrix, it suffices to design $\boldsymbol{k}_{d o}$ to be a constant vector that makes all eigenvalues of the composite-matrix $\left[\boldsymbol{D}_{o}+\boldsymbol{k}_{d o} \boldsymbol{h}_{o}\right]$ lie sufficiently-deep in the left-half of the complex-plane.

\section{Relation Between the $\left\{C_{i}\right\}_{1}^{M}$ in (1) and the

$$
\left\{z_{f}\right\}_{1}^{p} \text { in (6) }
$$

In the routine practical applications of disturbance-accommodating control-theory, it is neither necessary nor beneficial to "know" the values of the $C_{i}$ in (1), thanks to the ability to express the best/optimal control-input (control-law) in terms of reliable, real-time observer- estimates of $\hat{z}_{z}(t)$ (and of plant states $\left.\hat{x}_{i}(t)\right)$. This fortunate "ability" is so important it is called the Principle of (Real-Time) Optimal Disturbance-Accommodation [7; pg. 21920]
However, since the spline-model (1) and the statemodel (6) both represent the same (scalar) disturbance time-behavior $w(t)$, it is clear that the values of the $C_{i}$ in (1) must be related-to the values of the state-variables $z_{j}$ in (6). In fact, if the values of the $\left\{z_{j}(t)\right\}_{1}^{\rho}, \rho \leq \mathrm{M}$, are known at any moment $t \in \Omega$, then (in principle) the corresponding values of the $\left\{C_{i}\right\}_{1}^{M}$ in (1), at that same moment, should be uniquely determined (recall the basis-functions $f_{i}(t)$ in (1) are assumed independent). In the LD case, as considered here, the $C_{i}$ are related to the $z_{j}$ by a set of coupled, linear algebraic equations; see [9; Eq. (5d)] and the Examples below.

This observation suggests a novel procedure for experimentally evaluating the real-time values of the $C_{i}$ in (1) from reliable, real-time state-estimates $\hat{z}_{j}(t)$ obtained from a disturbance-state observer - . if and when there is some "need" to know/monitor the real-time $C_{i}$-values in (1).

\section{What Happens if the Actual $w(t)$-Behavior Doesn't Conform to the Models (1), (6)?}

Many users of disturbance-accommodating control theory have "observed" that disturbance-state observers (both full-order and reduced-order; continuous-time type) seem to correctly estimate the actual disturbance-input $w(t)$ even when the actual $w(t)$ time-behavior (mildly) deviates from the modeled time-behavior embodied in the spline-model (1) and in the state-model (6). For instance, if (1), (6) are based-on the behavior-model: $w(t)=$ unknown constant $=C_{1}$, the corresponding observer (8) (with a sufficiently short "settling-time) produces real-time estimates $\hat{w}(t) \triangleq h_{o} \hat{z}(t)$ that are surprisingly accurate even when the actual $w(t)$ is not constant but is (slowly) varying with time. The traditional intuitive explanation for this seemingly "adaptive/robustestimation behavior" is that a continuous-time disturbance-observer is continually re-evaluating, the state-estimate $\hat{z}(t)$, and reconciling $\hat{z}(t)$, with (1) based-on "current" actual $w(t)$-behavior and on the models (1), (6). Thus, in the case cited, a slowlyvarying actual $w(t)$ is "seen/perceived" by a continuous-time disturbance-state observer as a series of (infinitesimally) short, "constant" stair-step variations in the expected $w(t)=$ constant behavior embodied in (1), (6) and in the corresponding observer D-matrix structure.

In the remaining sections of this paper we will experimentally "confirm" this intuition by means of several worked examples using, realistic "compositestate" (plant + disturbance) observers [8; pp. 431437], and corresponding numerical, simulationresults. In particular, we will use the algebraic 
equations relating the $C_{i}$ in (1) to the $z_{i}$ in (4)-(6) to experimentally evaluate the disturbance observer's "perception" (re-construction) of the actual, real-time values of the "weighting-constants" $C_{i}$ in (1) when the actual $w(t)$ time-behavior $(t \in \Omega$ ) deviates (both "mildly" and "grossly"!) from the time-behavior modeled by (1) and embodied in the structure of the disturbance observer's D-matrix in (8). These results demonstrate that when the actual $w(t)$-behavior deviates from the behavior modeled by (1), the stateestimate $\hat{z}(t)$, generated by a continuous-time "disturbance-state observer" infers that the "constants" $C_{i}$ in (1) are rapidly changing-value in a virtually-continuous, stair-step manner, so that the resulting $w(t)$ in (1) then closely "matches" the actual $w(t)$-behavior.

\section{Illustrative Examples}

To demonstrate the apparent "adaptive-behavior" exhibited by disturbance-observers a simple pure inertia plant will be modeled with a disturbance applied. The system layout with a composite observer is given in Fig. 1.

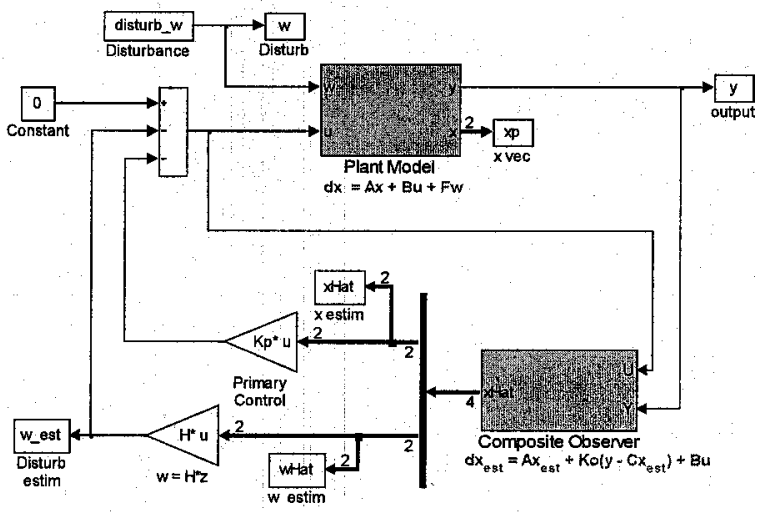

Figure 1: Overview of the Simulation

The state-space representation of the plant model is:

$$
\begin{aligned}
& A_{P}=\left[\begin{array}{ll}
0 & 1 \\
0 & 0
\end{array}\right] \quad B_{P}=\left[\begin{array}{l}
0 \\
1
\end{array}\right] \quad F=\left[\begin{array}{l}
0 \\
1
\end{array}\right] C=\lfloor 1,0\rfloor \\
& u=u_{s}+u_{d} ; u_{d} \text { - cancels w(t) effects. }
\end{aligned}
$$

State feedback control $u_{s}=K_{p} \hat{x}$ is achieved by multiplying the state estimate $\hat{x}$ by the gain $K p$. Disturbance cancellation is achieved by feeding back the disturbance estimate $\hat{w}$ to achieve $B_{p} u_{d} \approx-F w$. The the closed loop roots of the system at are placed at $\left[\begin{array}{ll}-2 & -2.5\end{array}\right]$. This results in $K p=\left[\begin{array}{ll}5 & 4.5\end{array}\right]$ This value of $K p$ will be used throughout the examples presented below.

To demonstrate the "adaptive" behavior of the disturbance observer we will first examine the performance of the disturbance observer when an appropriate dynamic basis for the observer is chosen when a "poor" choice is made for the dynamic basis of that observer. Let us first consider an example where the basis chosen for the disturbance observer is a "good" one ("good" in the sense that it contains a basis representative of the disturbance).

\section{Example \#1:}

- Disturbance a constant with a step-change at $\mathrm{t}=3 \mathrm{~s}$.

- Spline-model for the disturbance observer is $w(t)=c_{1}+c_{2} t$ (a "good" model)

The disturbance state model is:

$$
D=\left[\begin{array}{ll}
0 & 1 \\
0 & 0
\end{array}\right] \quad H=[1,0]
$$

Placing the composite observer poles [8; p.4-31] at $-6.6 \pm 2.4 i$ and $-6.9 \pm 1.2 i$ results in the following composite observer feedback gain:

$$
K o=\operatorname{col}(26.9,279,1320,2400)
$$

The results of this example are shown in Fig. 2.
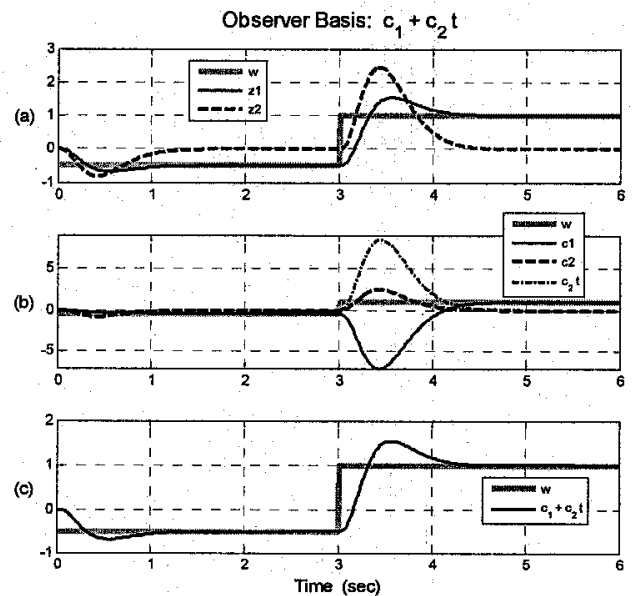

Fig. 2: Constant Disturbance, $c_{1}+c_{2} t$ Basis The disturbance $w(t)$ has an initial value of -0.5 and at $3 \mathrm{sec}$ jumps to a value of 1.0. As one might expect (since the observer dynamic basis is well chosen for this disturbance) the composite observer does a good job of estimating the disturbance and the observer identifies the $c_{1}, c_{2}$ as "stepwise-constants". When an unpredictable jump occurs at $t=3$ sec the observer experiences a transient but quickly recovers and locks back onto the disturbance $w(t)$. In Fig. 2 (a) we see the disturbance state $z(t)$. Since $H=\left[\begin{array}{ll}1 & 0\end{array}\right]$ the disturbance estimate $\hat{w}(t)=\hat{z}_{1}(t)(6 \mathrm{a})$. Examination of $z_{l}(t)$ in Fig. 2 (a) shows the good tracking of the 
disturbance $w(t)$ spoken of above. Additional insight can be gained by looking at the observer performance in terms of weighting coefficients in (1) for the spline function $w(t)=c_{1}+c_{2} t$. It is simple to solve for $c_{1}$ and $c_{2}$ in this example (this is not always so easy):

$$
\begin{aligned}
& c_{1}=z_{1}-z_{2} t \\
& c_{2}=z_{2}
\end{aligned}
$$

Fig. 2(b) shows that the $c_{2}$ coefficient has a significant role during the transient and then returns to zero as the observer locks back onto the constant disturbance and only $c_{l}$ is needed. Fig. 2 (c) confirms that the spline model of the disturbance $\hat{w}(t)=c_{1}(t)+c_{2}(t) t \quad$ is identical to the disturbance-state representation $\hat{w}(t)=\hat{z}_{1}(t)$.

\section{Example \#2}

- Disturbance is a sinusoid with a frequency of $0.75 \mathrm{rad} / \mathrm{sec}$ and an amplitude of 1.0 .

- Basis for the disturbance observer is $w(t)=c_{1}+c_{2} t$

The disturbance state model is the same as in example \#1 (11). The observer poles are also at the same location so the observer gain $K o$ is the same (12). This observer has the same basis so the relation between $z(t)$ and $c(t)$ is also the same (13).
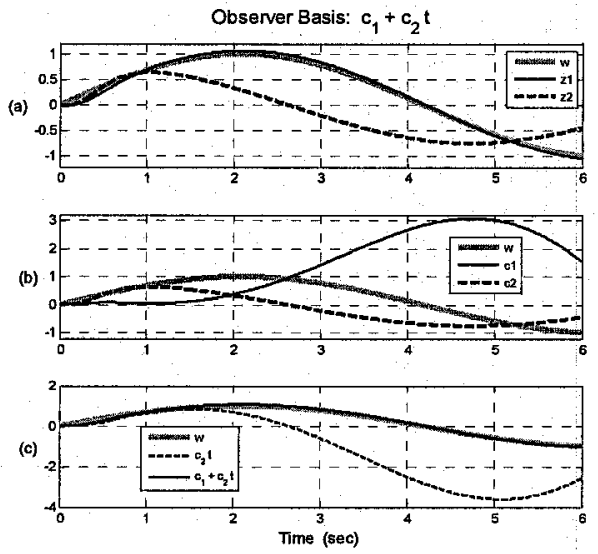

Fig. 3: Sinusoid Disturbance, $c_{1}+c_{2} t$ Basis

The surprising result shown in Fig. 3 is that a disturbance observer designed with a dynamic model ("internal-copy") for a constant plus a ramp is able to track a sinusoid quite well. Both $c_{1}$ and $c_{2}$ continually-adapt their "constant-values" to maintain accurate tracking of the disturbance.

Example \#3:

- Disturbance a constant with a step at $t=3 \mathrm{~s}$.
- Spline-Model for the disturbance observer is $w(t)=c_{1} \sin (\omega t)+c_{2} \cos (\omega t$

The disturbance state model defined in (6) for this disturbance basis is:

$$
D=\left[\begin{array}{cc}
0 & 1 \\
-\omega^{2} & 0
\end{array}\right] \quad H=\left[\begin{array}{ll}
\omega & 0
\end{array}\right]
$$

Composite observer poles at $-6.6 \pm 2.4 i$ and $6.9 \pm 1.2 i$ result in an observer feedback gain of:

$$
K o=\operatorname{col}(26.9,279,1740,2990) .
$$

The relationships between the disturbance-state variables $z(t)$ and the spline coefficients $c(t)$ for this observer are:

$$
\begin{aligned}
& c_{2}=\omega z_{1} \cos (\omega t)-z_{2} \sin (\omega t) \\
& c_{1}=\left(\omega z_{1}-c_{2} \cos (\omega t)\right) / \sin (\omega t) .
\end{aligned}
$$

In this example we are tracking a constant disturbance (with a jump) using an observer designed for the spline-model $c_{I} \sin (\omega t)+c_{2} \cos (\omega t)$. One would expect poor disturbance tracking but the results shown in Fig. 4 demonstrate surprisingly good tracking performance.
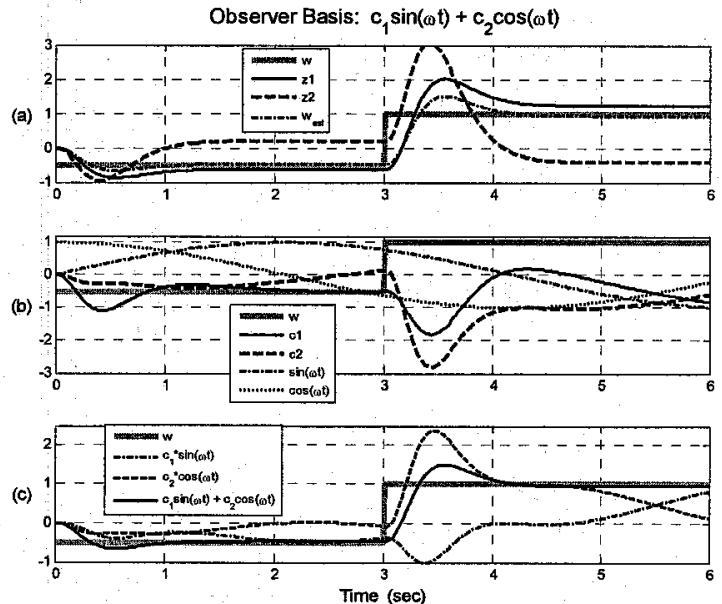

Fig. 4: Constant Disturbance, Sinusoidal Basis

Note that in Fig. 4 the values of $c_{I}$ and $c_{2}$ are constantly shifting value/"adapting" as the sine and cosine rise and fall with time " $\mathrm{t}$ ". The behavior can be explained by the fact that the disturbance observer constantly attempts to minimize the error between the disturbance estimate $\hat{w}(t)$ and the actual disturbance $w(t)(8)$. Even if the basis of the observer dynamics in no way reflects the dynamics of the actual 
disturbance the inherent property of a state-observer is that it will, according to the error the dynamics in (9), seek to minimize the error between the disturbance and its estimate. This property of an observer gives a control system gives what can be portrayed as "disturbance-adaptive" behavior.

There are limitations to how well an observer can track a disturbance with a "poor" selection of the observer basis dynamics. The performance of a sinusoid based disturbance-observer deteriorates as the frequency $\omega$ used in the disturbance-state model (14) becomes large relative to the frequency of jumps in the "constant" type disturbance. Similarly, the performance using a constant plus ramp basis for an observer tracking a sinusoid deteriorates as the frequency of the sinusoidal disturbance increases. This can be offset somewhat by placing the observer poles deeper into the left-half-plane.

\section{Conclusions}

The tracking performance of disturbance-observers when the internal-copy of $w(t)$ in the observer is not a good representation of a given disturbance dynamics has been studied. The ability of a disturbanceobserver to track a disturbance with dynamics completely different than what was assumed by the observer designer is the somewhat surprising result that this paper documents. The beauty of using a disturbance-observer in the control scheme is that even with totally unanticipated disturbance dynamics the "adaptive" capabilities inherent in the observer can still yield good results. This observer "adaptive" feature is not universal (the control designer should always try to include all important disturbance dynamics in the disturbance-observer basis) but it does account for some of the robustness that characterizes control systems that use a disturbanceobserver to accommodate disturbance inputs.

Acknowledgement: Thanks to Dr. C.D. Johnson for his assistance in the preparation of this paper.

\section{References Cited}

[1] Final Report (for the period June 1966-May 1967), NASA Contract \#NAS8-18008/Convair Div. of General Dynamics, Huntsville Office, Rpt. No. GDC DDF67-003 (Control No. DCN 16-75-00116, SI (IF)), Author: C. D. Johnson, dated May, 1967; Chapt. 3 "Optimal Control of the Disturbed Linear Regulator", pp. 49-67.
[2] Final Report (for the period July 1968-Aug. 1969), NASA Contract \#NAS8-21454/Convair Div. of General Dynamics, Huntsville Office, Rpt. No. GDC DDF68-005 (Control No. DCN 18-75-00086, (1F) and S-1, Author: C.D. Johnson, Dated Aug. 1969; Chapt. 4, "A Theory of Disturbance-Absorption for Dynamical Systems", pp. 68-92.

[3] Johnson, C. D., "Optimal Control of the Linear Regulator with Constant Disturbances," IEEE Trans. on Automatic Control, Vol. AC-13, No. 4, pp. 416-421, August 1968.

[4] Johnson, C. D., "Further Comments on 'Optimal Control of the Linear Regulator with Constant Disturbances,"' IEEE Trans. on Automatic Control, Vol. AC-15, No. 4, pp. 516-518, August 1970.

[5] Johnson, C. D., "Further Study of the Linear Regulator with Disturbances: The Case of Vector Disturbances Satisfying a Linear Differential Equation," IEEE Trans. on Automatic Control, Vol. AC-15, No. 2, pp. 222228, April 1970.

[6] Johnson, C. D., "Accommodation of Disturbances in Linear Regulator and Servo-Mechanism Problems," IEEE Trans. on Automatic Control, (Special Issue on the "Linear- QuadraticGaussian Problem"), Vol. AC-16, No. 6, pp. 635-644, December 1971. (invited paper)

[7] Johnson, C. D., "Accommodation of Disturbances in Optimal Control Problems," International Journal of Control, Vol. 15, No. 2, pp. 209-231, 1972. Also Proc. Third Southeastern Symposium on System Theory, Atlanta, GA, April 1971.

[8] Johnson, C. D., "Theory of Disturbance Accommodating Controllers," Chapter 7 in the book: Advances in Control and Dynamic Systems, Vol. 12, Edited by C. T. Leondes, Academic Press, 1976. [Translated into Russian, 1978]

[9] C. D. Johnson, "On the Theory of Discrete-Time Signals of the Discrete/Continuous Type," Proc. of the 35th Southeastern Symposium on System Theory, W. Virginia University, Morgantown, WV, pp. 113-121, March (2003). 


\title{
On the Observed Robustness of Disturbance-Observers; A Technical Explanation and Simulation Validation
}

\author{
Michael R. Hannan \\ NASA Marshall Space Flight Center \\ MS: EV41 \\ Huntsville, Alabama
}




\section{Introduction}

- A disturbance observer is a mathematical tool used to estimate in real-time the state of a disturbance acting on the system.

- With knowledge of the disturbance state a controller can compensate (accommodate) for the disturbance.

- We know that when the math model for the anticipated disturbance is a "good" one that a disturbance observer can quickly acquire and track the disturbance.

- It has been observed, however, that these observers display unexpected robustness when the functions chosen as the basis set for the observer are a poor reflection of the disturbance waveform. This robustness is what this presentation will illuminate and try to explain. 


\section{Background}

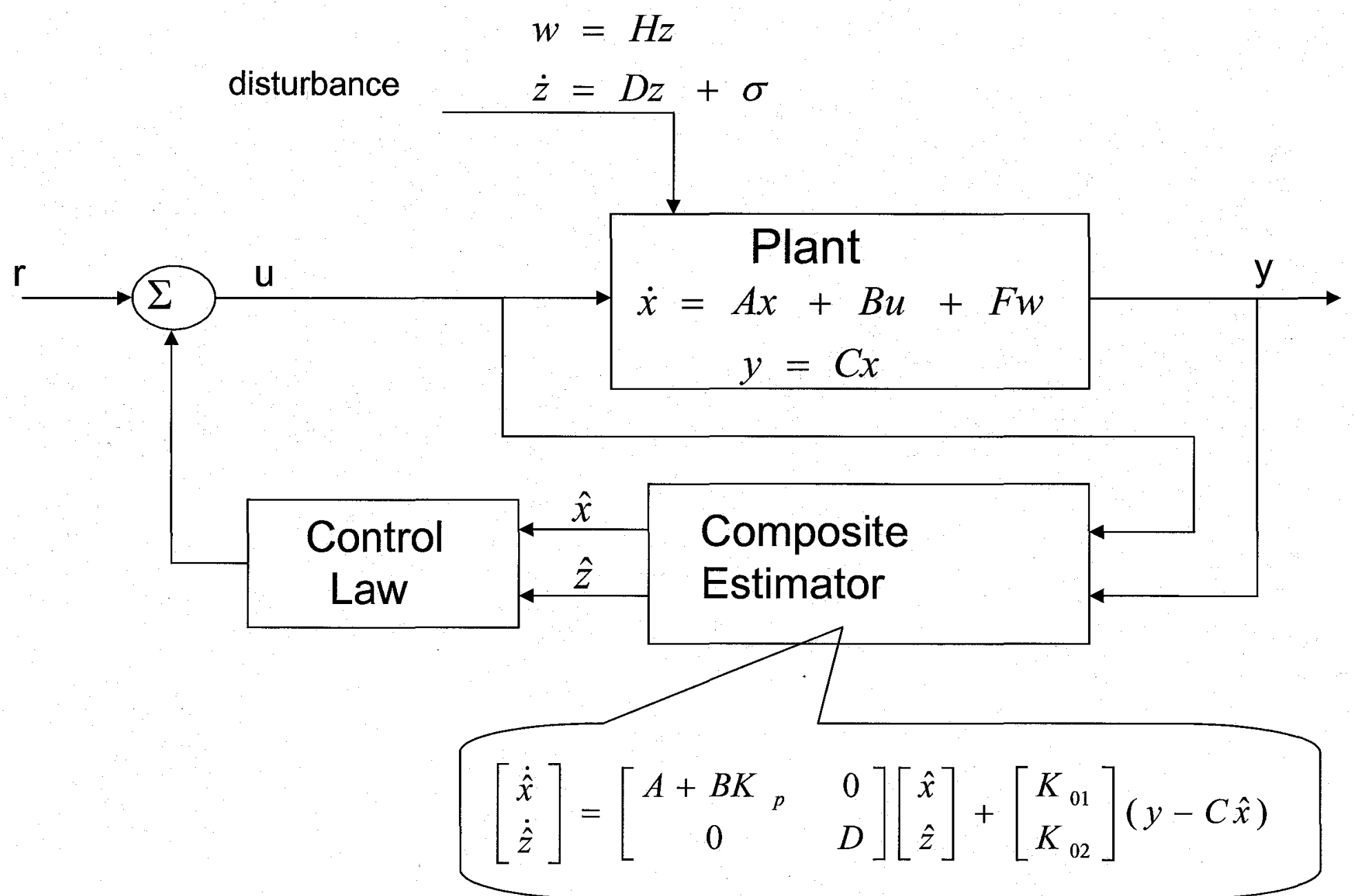




\section{Background}

- Many disturbances have waveform structure with once-in-a-while jumps

- As a the discontinuous jumps in a disturbance occur faster \& faster it approaches "noise" and statistical methods should be used.

- Many real world disturbances have waveform structure with only occasional jumps and can readily be estimated with a disturbance state observer

- A composite observer estimates both the plant state and the disturbance state

- To estimate the disturbance state we must first decide on an appropriate disturbance model. Common disturbance state models include:

- Constant

- Constant + ramp

- Sinusoid

- Pulse

- Some combination of the above 


\section{Waveform Model of a Disturbance}

- We must first mathematically model the anticipated disturbance " $w$ "

$$
w(t)=c_{1} f_{1}(t)+c_{2} f_{2}(t)+\cdots+c_{M} f_{M}(t)
$$

- The $f_{i}(t)$ are known independent functions of time

- The $c_{i}$ are unknown weighting coefficients

- This is referred to as the function space representation of $w(t)$

- This set of functions $f_{i}(t)$ form the basis-set and is chosen to reflect the expected waveform patterns of the disturbance

- The waveform model (1) is the mathematical foundation for this approach to disturbance modeling

- The waveform representation, however, can not be readily implemented into a control algorithm 


\section{Disturbance State Models}

- In order to use a disturbance observer one must first go to an alternative representation

- This alternative to a waveform representation is a disturbance state model

- Determining a disturbance state model can be approached as a reverse differential equations problem. The waveform representation in (1) can also be represented as the solution to the following general DE

$$
\frac{d^{\rho} w}{d t^{\rho}}+\alpha_{\rho} \frac{d^{\rho-1} w}{d t^{\rho-1}}+\cdots+\alpha_{2} \frac{d w}{d t}+\alpha_{1} w=0
$$

- The $\alpha_{i}$ in (2) are known parameters that depend only on the basis set $f_{i}(t)$

- Equation (2) can be written in the vector-matrix format:

$$
\begin{aligned}
& w(t)=H z \\
& \dot{z}=D z+\sigma(t)
\end{aligned}
$$

The $\sigma(t)$ term represents a series of dirac-impulses having unknown intensity and unknown arrival time.

This unknowable term is not part of the design but is included for mathematical completeness. 


\section{Example Disturbance Model Representation}

- For example, let us construct a disturbance state model for a disturbance that is mostly constant with occasional jumps

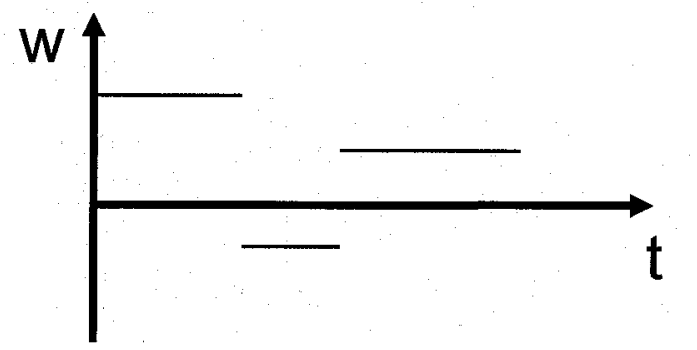

A disturbance $w(t)$ that is constant with "occasional" jumps

Waveform Representation: $w(t)=c_{1}$

Disturbance State Representation: $\quad \frac{d w}{d t}=0$

$$
H=[1], \quad D=[0]
$$




\section{Example Disturbance Model Representation}

- For a periodic disturbance with changing amplitude but a fixed frequency calls for a sinusoid basis function

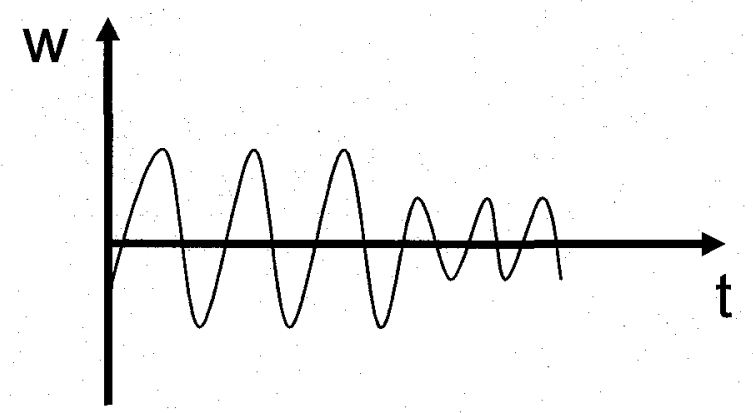

A disturbance $w(t)$ that is periodic

with changes in amplitude

Waveform Representation: $w(t)=c_{1} \sin \omega t+c_{2} \cos \omega t$

Disturbance State Representation: $\frac{d^{2} w}{d t^{2}}+\alpha_{1} w=0$

$$
H=\left[\begin{array}{ll}
\omega & 0
\end{array}\right], \quad D=\left[\begin{array}{cc}
0 & 1 \\
-\omega^{2} & 0
\end{array}\right]
$$




\section{Simulation Example}

- Will use the control of a pure inertia system as our test-bed

- Will examine the performance of the disturbance observer for "good" and "bad" choices of basis functions

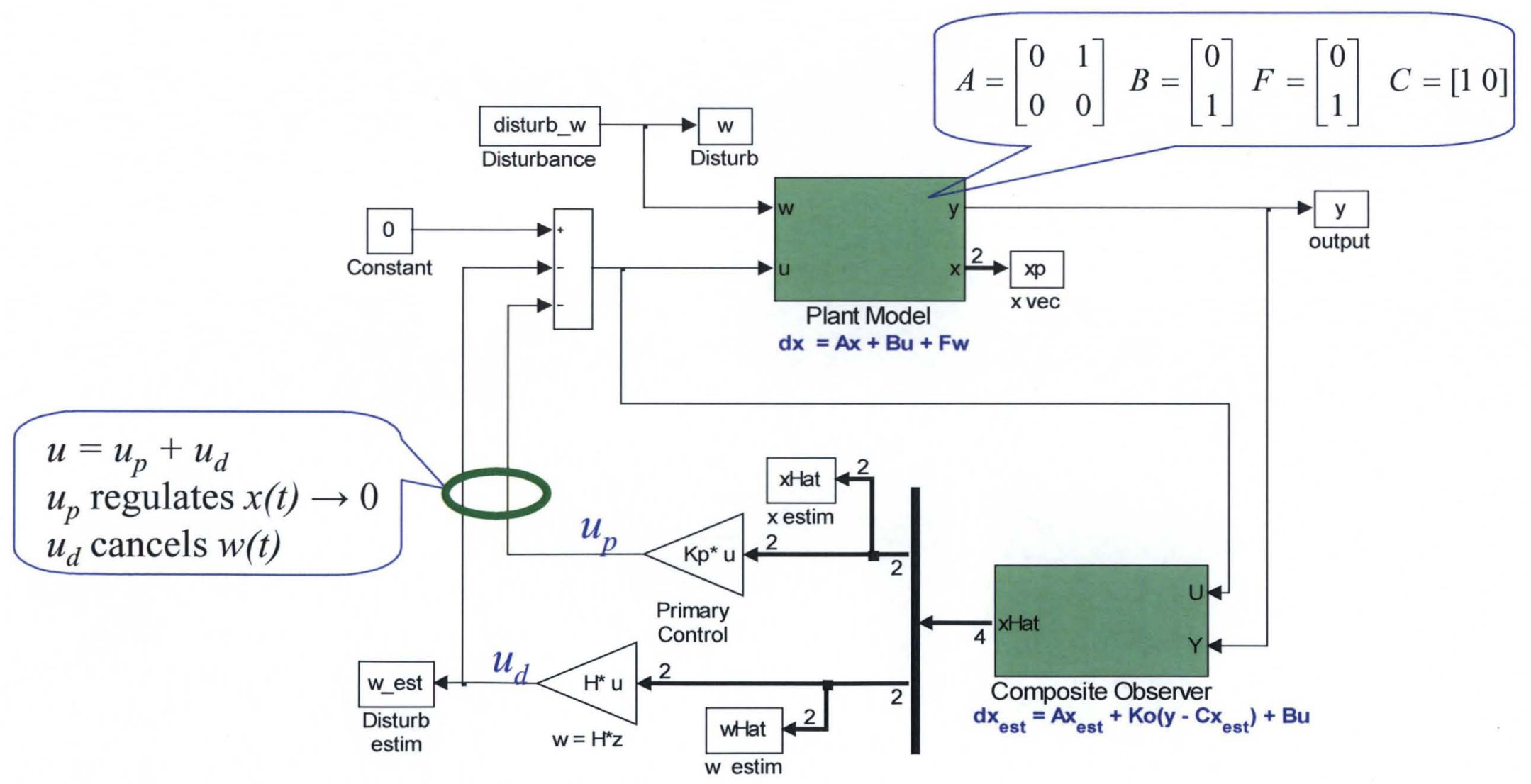




\section{Example 1}

- Disturbance is a constant with an unexpected jump

- Disturbance observer: "constant + ramp" basis (a "good" choice)

$$
\text { Disturbance Model: } w(t)=c_{1}+c_{2} t \Rightarrow D=\left[\begin{array}{ll}
0 & 1 \\
0 & 0
\end{array}\right] H=\left[\begin{array}{ll}
1 & 0
\end{array}\right]
$$

(a)

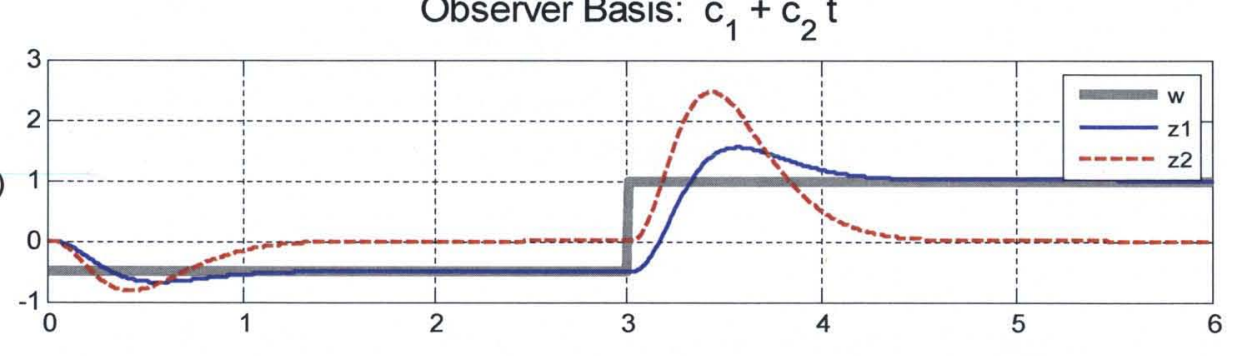

(b)

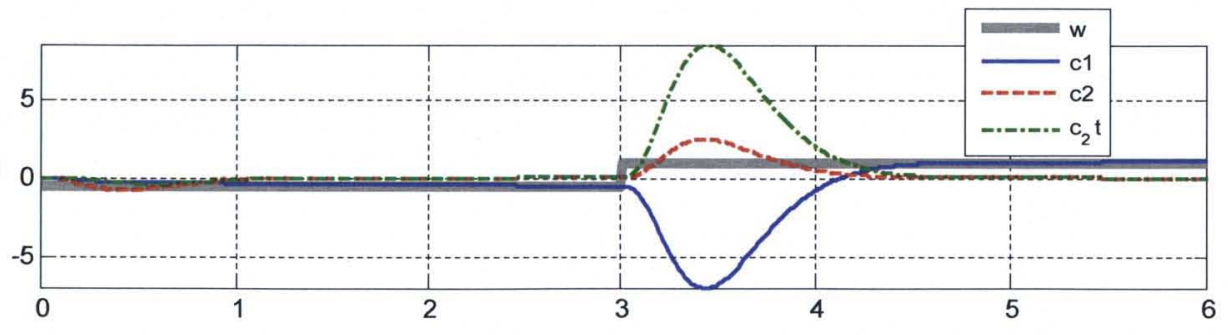

(c)

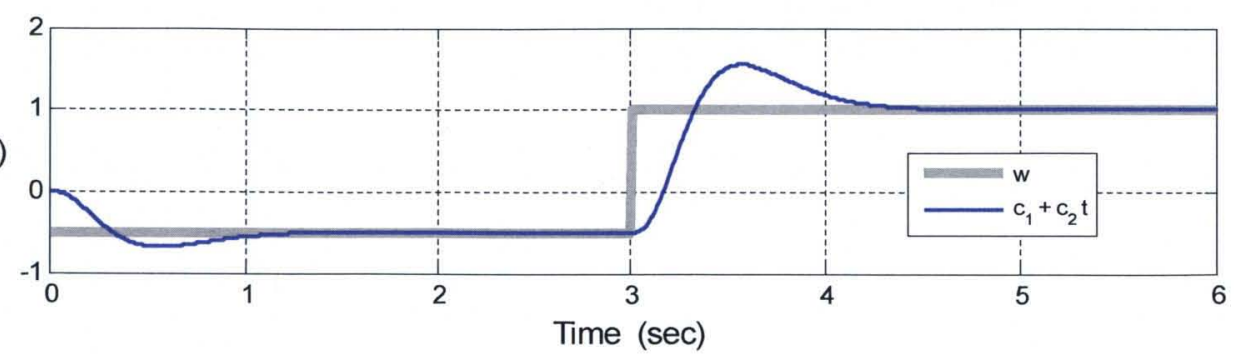

With a constant in the disturbance observer basis set one would expect the good tracking seen in the Figure 


\section{Example 2}

- Disturbance is a sinusoid ( $\omega=0.75 \mathrm{rad} / \mathrm{s})$

- Disturbance observer: "constant + ramp" basis (a "poor" choice)

Disturbance Model: $w(t)=c_{1}+c_{2} t \Rightarrow D=\left[\begin{array}{ll}0 & 1 \\ 0 & 0\end{array}\right] H=\left[\begin{array}{ll}1 & 0\end{array}\right]$

Observer Basis: $c_{1}+c_{2} t$

(a)

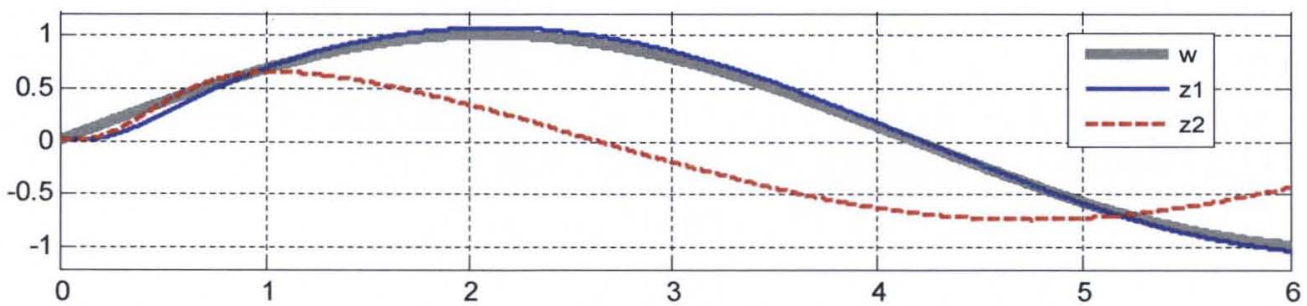

(b)

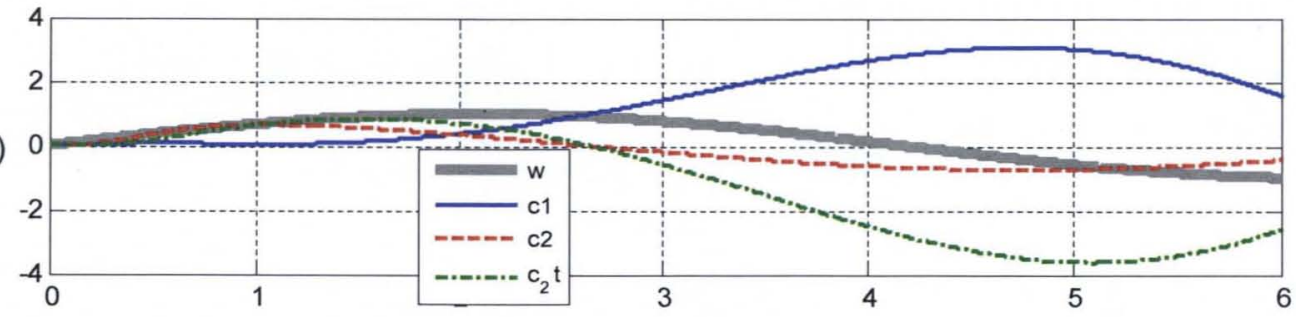

The observer tracks the sinusoid disturbance using a constant + ramp internal model for the disturbance. Note how $\mathrm{C}_{1} \& \mathrm{C}_{2}$ constantly adjust to minimize the error between the disturbance and its estimate

(c)

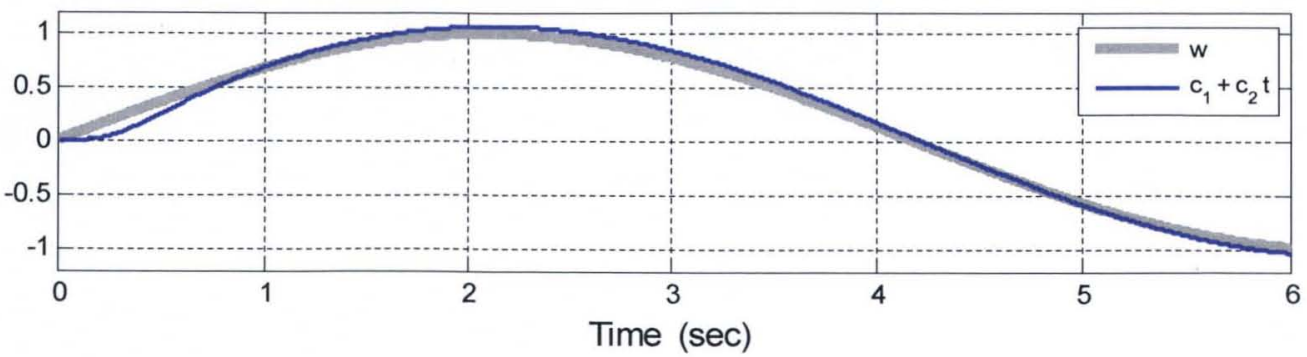




\section{Example 3}

- Disturbance is a constant with an unexpected jump

- Disturbance observer: Sinusoid basis (a "poor" choice)

Disturbance Model: $w(t)=c_{1} \sin \omega t+c_{2} \cos \omega t \Rightarrow D=\left[\begin{array}{cc}0 & 1 \\ -\omega^{2} & 0\end{array}\right] H=\left[\begin{array}{ll}\omega & 0\end{array}\right]$

Observer Basis: $c_{1} \sin (\omega t)+c_{2} \cos (\omega t)$

(a)

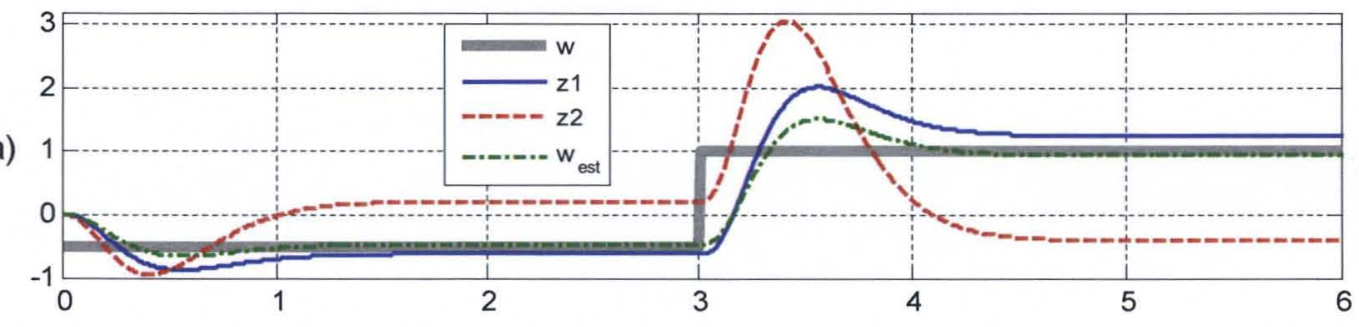

An observer designed for a sinusoid disturbance successfully tracks a constant disturbance! The coeffs $\mathrm{c}_{1} \& \mathrm{C}_{2}$ are rapidly changing but the net disturbance estimate $(\hat{w})$ smoothly \& accurately

(b)

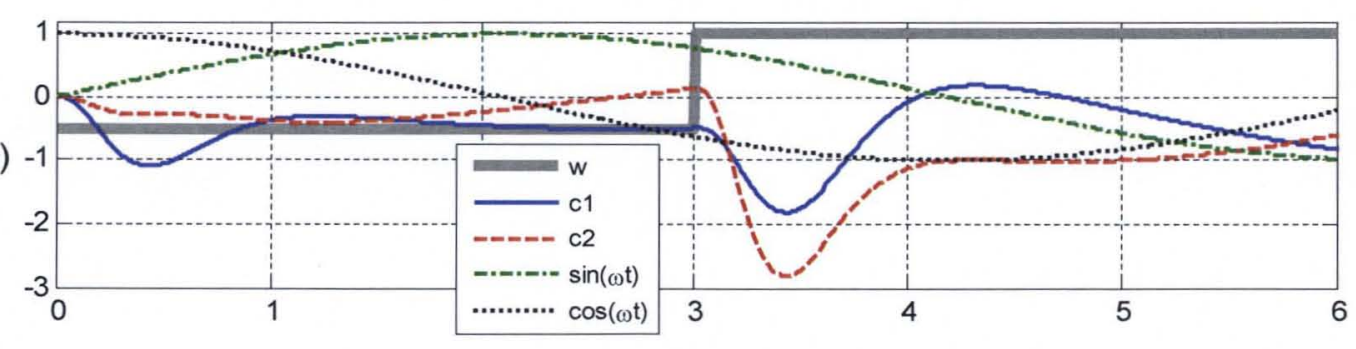
tracks the disturbance

(c)

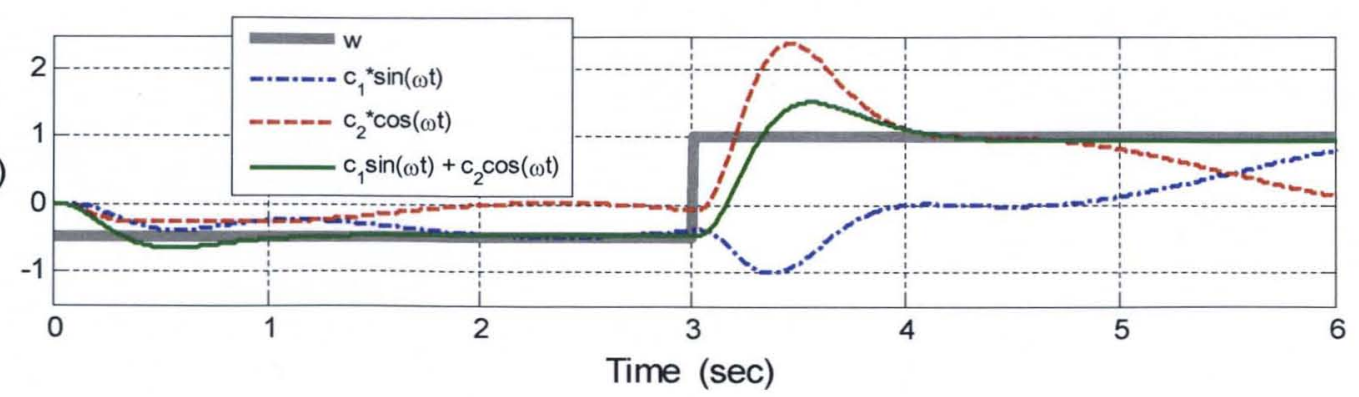




\section{Conclusions}

- Disturbance observers demonstrate an ability to "adapt" and successfully track a disturbance with dynamics completely different from what the observer was designed for

- There are limits to this adaptability. It is always best to pick a basis-set that is representative of the expected disturbance dynamics

- The ability of an observer designed for a sinusoid to track a constant with jumps deteriorates as the frequency of the sinusoid basis becomes large relative to the frequency of the jumps

- Similarly, the performance of a disturbance observer with a constant basis function deteriorates as the frequency of the sinusoid disturbance increases

- An observer is a closed loop subsystem that is structured to drive an estimate to zero. As a result of this disturbance observers will make every effort to adjust internal coefficients so that the net error is minimized. This effect manifests itself as the "adaptive" capability of a disturbance observer to track disturbances very different from what it was originally designed for. 


\section{Backup}




\section{Derivation of the function-space Coefficients}

- The disturbance-state $z$ is readily available from the observer. Much insight can be gained by examining the dynamics in the function space.

- The disturbance state $z$ can be converted to the $c_{i}$ values in (1) needed to represent $w(t)$ in the wavefrom model

- Disturbance observer with a "constant + ramp" basis

$$
z_{1}=w=c_{1}+c_{2} t \quad \text { and } \quad z_{2}=\dot{z}_{1}=\frac{d}{d t}\left(c_{1}+c_{2} t\right)=c_{2}
$$

Therefore $\begin{aligned} & c_{1}=z_{1}-z_{2} t \\ & c_{2}=z_{2}\end{aligned}$ 


\section{Derivation of the function-space Coefficients}

- Disturbance observer with a "sinusoid" basis

- Recall the waveform model is $w(t)=c_{1} \sin \omega t+c_{2} \cos \omega t$

- The state model is:

$$
w(t)=H z, \dot{z}=D z \Rightarrow H=\left[\begin{array}{ll}
\omega & 0
\end{array}\right], \quad D=\left[\begin{array}{cc}
0 & 1 \\
-\omega^{2} & 0
\end{array}\right]
$$

Start with: $\quad w(t)=\omega z_{1}=c_{1} \sin \omega t+c_{2} \cos \omega t$

$$
z_{1}=\frac{c_{1}}{\omega} \sin \omega t+\frac{c_{2}}{\omega} \cos \omega t
$$

Take the derivative of $z_{1} \rightarrow \quad \dot{z}_{1}=z_{2}=c_{1} \cos \omega t-c_{2} \sin \omega t$

Have 2 equations \& two unknowns. Solving we find that

$$
\begin{aligned}
& c_{2}=\omega z_{1} \cos (\omega t)-z_{2} \sin (\omega t) \\
& c_{1}=\left(\omega z_{1}-c_{2} \cos (\omega t)\right) / \sin (\omega t) .
\end{aligned}
$$




\section{Example B1}

- Disturbance is a sinusoid

- Disturbance observer: Sinusoid basis (a "good" choice)

Disturbance Model : $w(t)=c_{1} \sin \omega t+c_{2} \cos \omega t \Rightarrow D=\left[\begin{array}{cc}0 & 1 \\ -\omega^{2} & 0\end{array}\right] H=\left[\begin{array}{ll}\omega & 0\end{array}\right]$

(a)

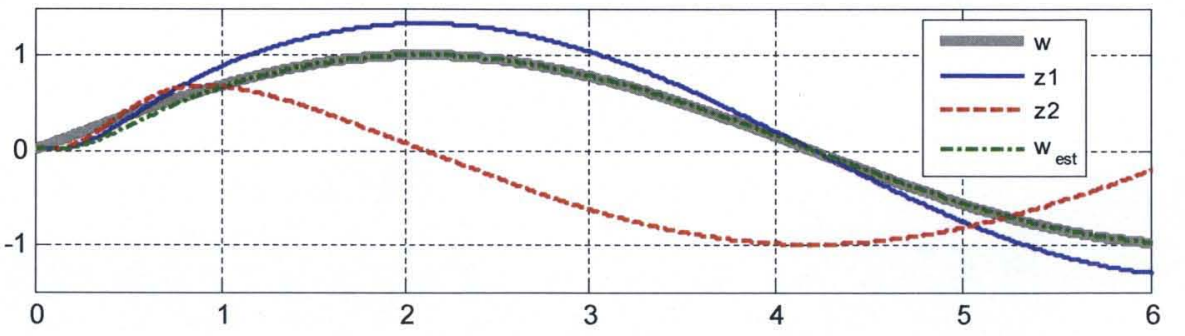

(b)

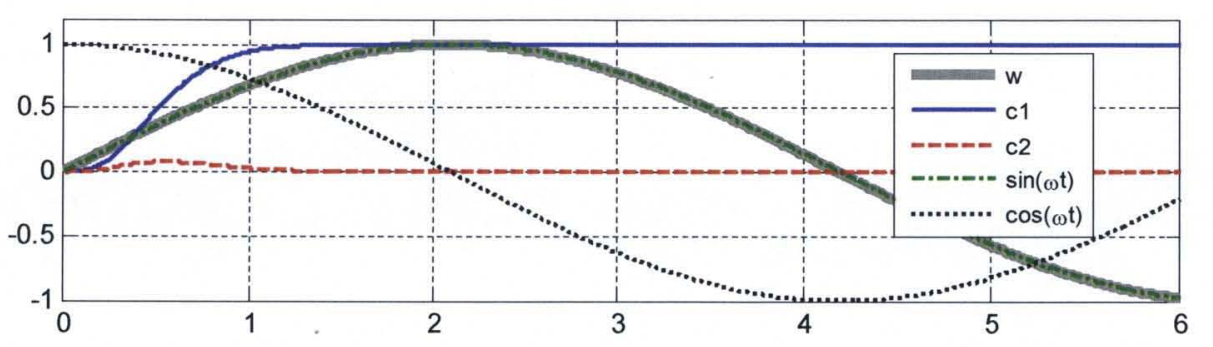

The disturbance is a pure sine with no phase angle. As one might expect the $\mathrm{C}_{1}$ coeff goes to 1.0 and the $\mathrm{C}_{2}$ coeff goes to zero after the initial startup transient. There is a startup transient because the initial conditions of the observer integrators are set to zero.

(c)

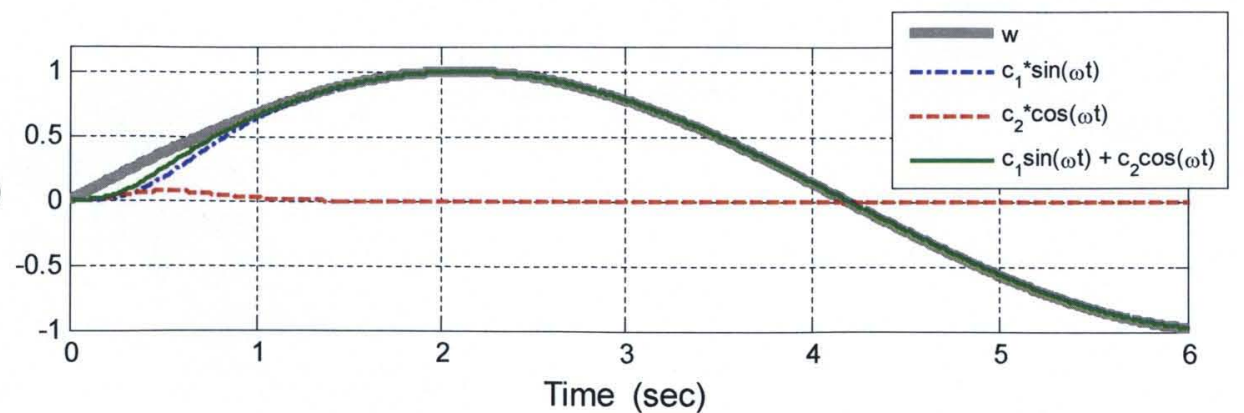

